\title{
PROSPECT
}

\section{Chromatin Remodeling, Histone Modifications, and DNA Methylation-How Does it All Fit Together?}

\author{
Theresa M. Geiman and Keith D. Robertson* \\ Epigenetic Gene Regulation and Cancer Section, National Cancer Institute, National Institutes of Health, \\ Bethesda, Maryland 20892
}

\begin{abstract}
DNA methylation is important in the control of gene transcription and chromatin structure. The complexities of this process are just beginning to be elucidated in relationship to other epigenetic mechanisms. Exciting new research in the areas of histone methylation and chromatin remodeling make it clear just how important the connections between these various mechanisms and DNA methylation are for the control of chromosome structure and gene expression. Emerging evidence suggests that chromatin remodeling enzymes and histone methylation are essential for proper DNA methylation patterns. Other histone modifications, such as acetylation and phosphorylation, in turn, affect histone methylation and histone methylation also appears to be highly reliant on chromatin remodeling enzymes. This review will summarize what is likely only the beginning of a flood of new information that will ultimately link all epigenetic modifications of the mammalian genome. A model will also be put forth to account for how chromatin modifications lead to genomic DNA methylation patterns. J. Cell. Biochem. 87: 117-125, $2002 . \quad$ Published 2002 Wiley-Liss, Inc.
\end{abstract}

Key words: epigenetics; DNA methylation; histone methylation; chromatin remodeling; gene expression

DNA methylation is an epigenetic process involved in controlling many cellular functions, such as gene expression, genomic stability, X chromosome inactivation, and chromatin structure [Baylin and Jones, 2002]. This process consists of the covalent addition of a methyl group to the 5-position of the cytosine base predominantly when it occurs in the context of CpG. DNA methylation occurs non-randomly in the genome, with certain regions such as repetitive and transposable elements being hypermethylated and transcriptionally inactive, while other regions such as $\mathrm{CpG}$ islands in promoters of house-keeping genes are hypomethylated

and transcriptionally competent [Baylin and

K.D. Robertson is a Cancer Scholar, and T.M. Geiman is a Pharmacology Research Associate Training Fellow.

Grant sponsor: NIH (to K.D.R.); Grant number: CA8453501; Grant sponsor: National Institute of General Medical Sciences, NIH (to T.M.G.).

*Correspondence to: Keith D. Robertson, Epigenetic Gene Regulation and Cancer Section, National Cancer Institute, National Institutes of Health, 41 Library Drive, Bldg. 41, Rm. C302, Bethesda, MD 20892.

E-mail: robertk@mail.nih.gov

Received 17 July 2002; Accepted 18 July 2002

DOI 10.1002/jcb.10286

Published 2002 Wiley-Liss, Inc. ${ }^{\dagger}$ This article is a US Government work, and as such, is in the public domain of the United States of America.
Jones, 2002]. Additionally, DNA methylation patterns change during development and differentiation. In particular, there is a dramatic demethylation of the genome that occurs after fertilization, followed by the de novo establishment of methylation patterns following implantation [Reik et al., 2001]. For this reason, it is essential to tightly regulate the establishment and maintenance of proper methylation patterns both temporally and spatially. In the instances in which DNA methylation goes awry, it is evident just how important and complex this process is. Disruption of proper DNA methylation occurs in several human disorders including ATRX, Fragile X, and ICF syndromes [Robertson, 2002]. Additionally, alterations in DNA methylation frequently occur in many human cancers with normally hypomethylated regions becoming methylated (such as $\mathrm{CpG}$ island promoters), and regions with high levels of methylation losing methyl groups (such as repetitive DNA and transposons) [Baylin and Jones, 2002]. This reversal of normal DNA methylation patterns can lead to alterations in gene expression and genome stability through changes in chromatin structure.

The process of DNA methylation in mammals is carried out by at least three catalytically active DNA methyltransferase (DNMT) enzy- 
mes. The mammalian family of DNMTs consists of five known members, DNMT1, DNMT2, DNMT3A, DNMT3B, and DNMT3L [Bestor, 2000]. While these five proteins are placed in the DNMT family based on sequence homology, only DNMT1, DNMT3A, and DNMT3B (Table I) have been shown to exhibit catalytic activity [Hsieh, 1999]. Of these three enzymes, DNMT3A and 3B are believed to be the primary de novo DNMTs that establish new methylation patterns [Okano et al., 1998; Aoki et al., 2001; Yokochi and Robertson, 2002]. These enzymes are probably responsible for the reestablishment of methylation patterns during embryogenesis. Since the process of de novo methylation is especially critical during embryonic development, the de novo DNMTs are highly expressed in embryonic cells [Okano et al., 1998]. In contrast, DNMT1 functions as the maintenance DNMT to ensure that the DNA methylation pattern is faithfully copied to the newly synthesized DNA strand following replication [Bestor, 2000; Yokochi and Robertson, 2002]. For this reason, DNMT1 is associated with replication foci during $\mathrm{S}$ phase of the cell cycle [Leonhardt et al., 1992]. These three DNMTs consist of a C-terminal catalytic region and an $\mathrm{N}$-terminal regulatory region. All three enzymes contain similar catalytic domains, while they diverge significantly in their regulatory regions, consistent with their differing functions [Okano et al., 1998]. Within the Nterminal regulatory region, DNMT3A and B are more similar to each other than to DNMT1. DNMT3A and 3B contain a 'PWWP' domain of unknown function, as well as a cysteine-rich ATRX-like plant homeodomain (PHD) region believed to mediate protein-protein interactions. The DNMT1 N-terminal domain contains a nuclear localization signal, a region for targeting to replication foci, and a different type of cysteine-rich region [Bestor, 2000].

The importance of these DNMTs in development has been clearly established by the generation of null mice as well as the study of the human ICF syndrome (Table I). DNMT1 null mice die early in embryonic development, and exhibit severe genomic hypomethylation $[\mathrm{Li}$ et al., 1992; Lei et al., 1996]. In contrast, DNMT3A null mice develop normally until birth but become runted and die by 4 weeks of age [Okano et al., 1999]. Homozygous deletion of DNMT3B, however, resulted in early embryonic lethality with a pronounced loss of genomic
DNA methylation in pericentromeric heterochromatin repeats [Okano et al., 1999]. This defect is similar to that seen in lymphocytes of ICF syndrome patients. In this disorder, which stands for Immune deficiency, Centromeric instability, and Facial anomalies, patients have immune defects that frequently lead to death at an early age. DNMT3B has been found to be mutated in the majority of cases of ICF syndrome that have been investigated [Hansen et al., 1999; Okano et al., 1999]. This partial loss of DNMT3B function leads to hypomethylation and chromatin decondensation of the repetitive satellite DNA in pericentromeric heterochromatin, as well as changes in gene expression [Ehrlich et al., 2001]. This indicates that a particular chromatin structure is essential to maintain proper gene expression patterns in mammalian cells and that DNA methylation is a critical player in this process.

\section{HISTONE/DNA METHYLATION CONNECTION}

Gene transcription in mammalian cells does not occur on naked DNA, but instead occurs in the context of chromatin (146 bp of DNA wrapped around a nucleosome composed of two molecules each of histones H2A, H2B, H3, and $\mathrm{H} 4)$. As a result, modifications to the chromatin and the histones themselves impact gene expression. The modification of DNA by the addition of a methyl group can alter the chromatin by leading to a more compact structure, and thus silencing gene expression. Recent studies indicate that there are other epigenetic processes such as histone modifications (particularly the N-terminal "tails") and chromatin remodeling that can cooperate to control chromatin structure and ultimately cellular processes such as gene expression and DNA methylation itself. Histone $\mathrm{H} 3$ and $\mathrm{H} 4$ tail modifications include acetylation, phosphorylation, ubiquitination, and methylation. While acetylation and phosphoylation of histone tails have been extensively researched, studies in recent years indicate that histone modifications other than acetylation and phosphorylation are also critical to the function of the chromatin in which they occur. In particular, methylation of lysine residues on the histone tails of $\mathrm{H} 3$ and $\mathrm{H} 4$ appears to provide an additional layer of control over the chromatin structure, and ultimately over gene expression. Histone lysine methyla- 


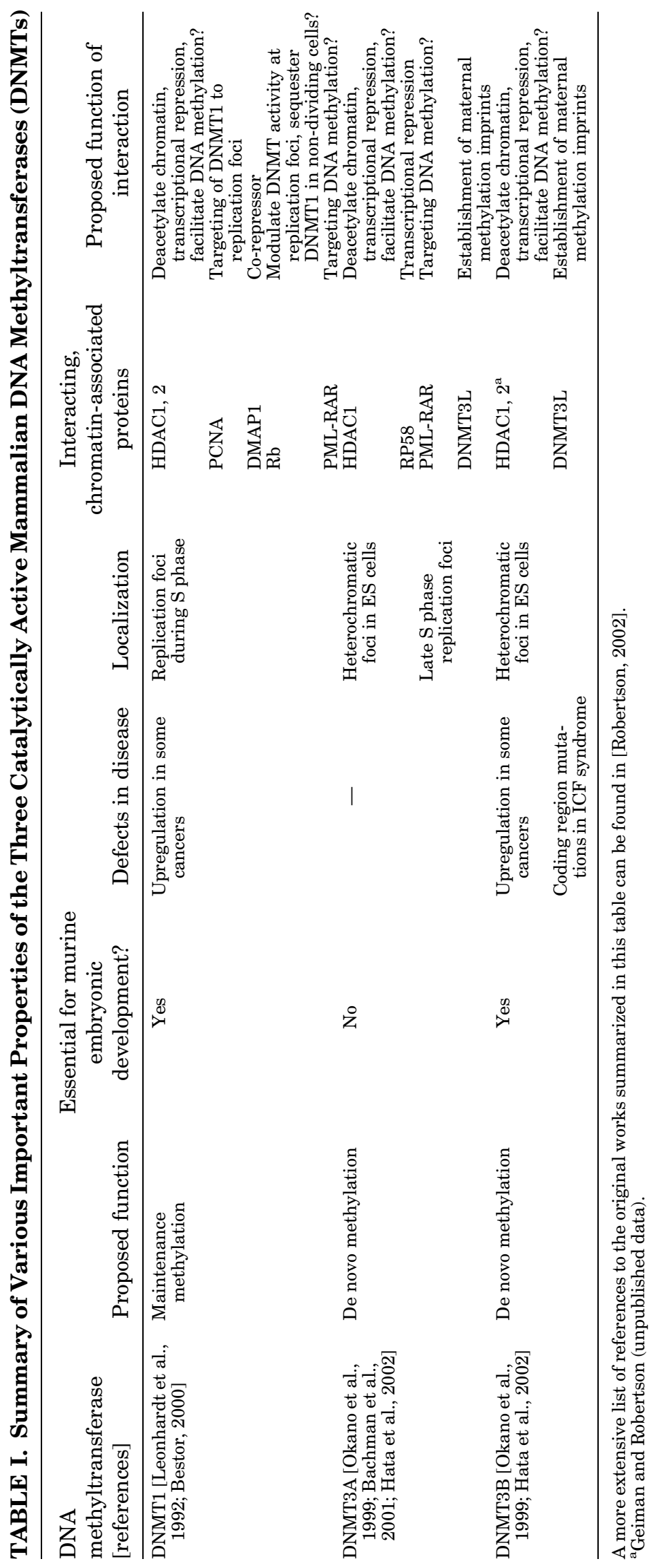


TABLE II. Summary of Known and Proposed Links Between DNA Methylation and Histone Modifications (Methylation and Acetylation) in the Three Most Extensively Studied Systems

\begin{tabular}{|c|c|c|c|c|}
\hline Organism & $\begin{array}{c}\text { SUV39H1-like } \\
\text { H3, K9 methylase }\end{array}$ & $\begin{array}{c}\text { DNA } \\
\text { methyltransferase }\end{array}$ & $\begin{array}{l}\text { "Targeting module" } \\
\text { recruiting the DNMT } \\
\text { to } \mathrm{H} 3, \mathrm{~K} 9 \text { methylated } \\
\text { region }\end{array}$ & $\begin{array}{l}\text { Associated HDACs and } \\
\text { their interaction partner(s) }\end{array}$ \\
\hline Arabidopsis & Kryptonite (KYP) & $\begin{array}{l}\text { Chromomethylase } 3 \\
\text { (CMT3) }\end{array}$ & $\mathrm{HP} 1 \beta$ & $\begin{array}{l}\text { Unknown, possibly } \\
\text { KYP-HDACx }\end{array}$ \\
\hline Neurospora & DIM5 & $\begin{array}{l}\text { Unknown, possibly } \\
\text { DIM2 }\end{array}$ & Unknown & Unknown \\
\hline Mammals & $\begin{array}{l}\text { Unknown, likely } \\
\text { SUV39H1 }\end{array}$ & $\begin{array}{l}\text { Unknown, likely } \\
\text { DNMT3A, } \\
\text { DNMT3B }\end{array}$ & $\begin{array}{l}\text { Unknown, likely an HP1 } \\
\text { family member }\end{array}$ & $\begin{array}{l}\text { DNMT1, 3A, 3B-HDAC1,2 } \\
\text { SUV39H1-HDAC1 }\end{array}$ \\
\hline
\end{tabular}

Future studies will fill-in the 'unknowns' in this table and will likely show that multiple epigenetic chromatin regulatory mechanisms are directly linked.

tion is catalyzed by a group of enzymes called histone methyltransferases (HMTase) [Lachner and Jenuwein, 2002]. Members of this enzyme family contain a conserved SET domain that is flanked by cysteine-rich regions [Rea et al., 2000]. There appears to be intricate interplay between different modifications on various sites of the histone tails of $\mathrm{H} 3$ and $\mathrm{H} 4$, some of which act antagonistically to regulate the conversion from an active chromatin state to an inactive one. In particular, methylation of lysine residues on the histone $\mathrm{H} 3$ tail seems to be intimately connected to the DNA methylation status of the particular chromatin region in which it occurs.

The connection between histone methylation and DNA methylation comes most convincingly from recent studies in Neurospora and Arabidopsis. In Neurospora, mutations in a histone methyltransferase gene (DIM-5), specific for histone H3 lysine 9, results in a complete loss of genomic DNA methylation in Neurospora [Tamaru and Selker, 2001]. Genomic DNA methylation is also decreased if wild type Neurospora histone H3 lysine 9 is replaced by $\mathrm{H} 3$ with an altered amino acid at position 9 that cannot be methylated [Tamaru and Selker, 2001]. Additionally, a partial loss of genomic DNA methylation was found when a related gene, kryptonite (KYP), was mutated in Arabidopsis [Jackson et al., 2002]. Interestingly, in Arabidopsis, the DNMT chromomethylase 3 (CMT3) was shown to interact with the methyl-lysine binding protein HP1 $\beta$, thus providing an elegant mechanism for targeting DNA methylation to regions of the genome containing histone methylation [Jackson et al., 2002]. These studies indicate that the establishment and maintenance of DNA methylation is depen- dent upon histone H3 lysine 9 methylation in both of these organisms and may be a common eukaryotic epigenetic mechanism (Table II). Cooperation between histone H3 lysine 9 methylation and DNA methylation is further supported by the fact that both modifications are generally associated with transcriptionally silent heterochromatin. Evidence for the involvement of histone methylation in the establishment of a repressive chromatin state with DNA methylation comes from the observation that histone $\mathrm{H} 3$ methylated at lysine 9 is a binding site for the heterochromatin protein HP1 [Bannister et al., 2001; Lachner et al., 2001]. Additionally, the histone H3 lysine 9 methyltransferase SUV39H1 colocalizes and interacts with HP1 in regions of heterochromatin [Lachner et al., 2001]. HP1 is a major component of heterochromatin and contributes to the establishment and maintenance of the transcriptionally silent state of heterochromatin. Interestingly, DNMT3 proteins also co-localize with HP1 at heterochromatic sites in embryonic stem cells (Table I) suggesting that DNA methylation may be targeted to heterochromatic sites through HP1 [Bachman et al., 2001]. The exact relationship between HP1 proteins, DNMT's, and HMTases in mammalian cells remains unclear (Table II), but is currently the subject of intensive research.

The interplay between various histone $\mathrm{H} 3$ tail modifications particularly on lysines 4,9 , and 14 as well as serine 10, appears to contribute to the control of the chromatin environment and thus the status of gene expression. As Figure 1 shows, an active transcriptional state is associated with phosphorylation of serine 10 that precedes and facilitates the acetylation of lysine 14 as well as the methylation of lysine 4 . 

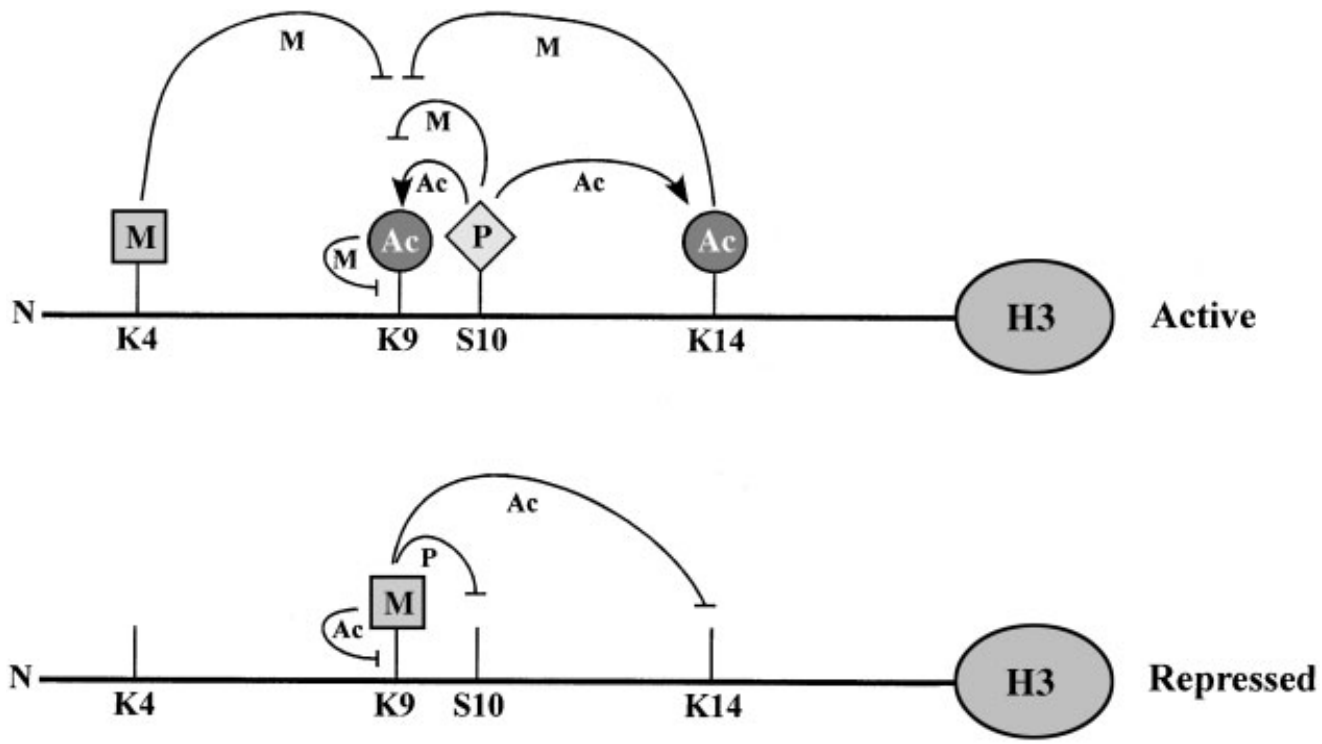

Fig. 1. Histone $\mathrm{H} 3 \mathrm{~N}$-terminal tail modifications in transcriptionally active or repressed chromatin. Arrows indicate one modification facilitating the occurrence of another modification (e.g., phosphorylation of serine 10 facilitates acetylation of K9 and K14). Blocked arrows indicate the inhibition of a modifica-

The methylation of lysine 4 is catalyzed by a different histone methyltransferase family member, SET9/SET7 [Wang et al., 2001]. Alternatively, a repressive transcriptional state is associated with deacetylation of lysines 9 and 14 , dephosphorylation of serine 10 , and methylation of lysine 9 . The phosphorylation of serine 10 appears to facilitate acetylation of lysine 9 and 14, which in turn prevents methylation of lysine 9 of histone H3 [Rea et al., 2000]. Acetylation of lysine 14 leads to an active gene status since this modification blocks the methylation of lysine 9 . Conversely, evidence from Drosophila and fission yeast indicate that histone deacetylases act to allow histone H3 lysine 9 specific methyltransferases to bind and methylate their target [Czermin et al., 2001; Nakayama et al., 2001]. In Drosophila cells, Su(VAR)3-9, the histone H3 lysine 9 methyltransferase homologous to mammalian SUV39H1, interacts with histone deacetylase to remove acetyl groups from neighboring residues [Czermin et al., 2001]. Additionally, evidence from fission yeast shows that a histone deacetylase specific for lysine 14 (Clr3) is necessary for Clr4, a homologue of the SUV39H1 histone methyltransferase, to methylate the $\mathrm{H} 3$ lysine 9 residue [Nakayama et al., 2001]. This group also found that the histone deacetylase Clr3 is necessary for the localization of the yeast tion by the presence of another modification. Modification abbreviations: $\mathrm{M}$, histone methylation; Ac, histone acetylation; $\mathrm{P}$, histone phosphorylation. Key amino acid residues subject to modification: K (lysine) 4, 9, and 14; S (serine) 10.

HP1 homologue to heterochromatin. It seems, therefore, that methylation of lysine 4 and lysine 9 of histone H3 are antagonistic modifications signifying opposing chromatin and gene expression states.

\section{CHROMATIN REMODELING AND DNA METHYLATION}

While DNA methylation and histone modifications are clearly important for the control of gene expression, it now appears that chromatin remodeling proteins also play a role in the regulation of this process. The SNF2 family of chromatin remodeling proteins acts in various cellular processes such as gene expression, replication, DNA repair, and recombination [Vignali et al., 2000; Havas et al., 2001]. SNF2 family members contain seven helicase domains but do not exhibit the classical strand separating activity of helicases. Instead, these proteins utilize ATP to alter the structure of chromatin through disruption of the histone/DNA contacts. In the context of gene expression, SNF2 family members are believed to function in transcriptional activation as well as repression depending on the SNF2 factor and the proteins with which it interacts. Recent evidence implicates several of these family members in 
the process of maintaining proper DNA methylation patterns in organisms as diverse as Arabidopsis thaliana and humans.

The first member of the SNF2 helicase/ ATPase family to be connected to DNA methylation was the A. thaliana protein DDM1 (decrease in DNA methylation) [Jeddeloh et al., 1999]. This gene, when mutated, results in a $70 \%$ reduction in global DNA methylation levels. The decrease in DNA methylation becomes more pronounced with successive generations. Repetitive elements are the first DNA sequences to exhibit a reduction in DNA methylation, and a decrease is only observed in low copy number genes following successive generations of self-pollination. This loss of DNA methylation must disrupt the formation of silent heterochromatin since expression and mobility of transposable elements is altered in DDM1 mutants [Miura et al., 2001]. The DDM1 gene is highly related to the mammalian lymphoid specific helicase (Lsh) (Hells, PASG) gene. $L s h$ was first identified as an SNF2 helicase family member highly expressed in fetal thymus and activated lymphocytes [Jarvis et al., 1996]. It has since been found to be expressed ubiquitously in embryonic tissues [Geiman et al., 2001], and has been linked to cell proliferation [Geiman and Muegge, 2000; Raabe et al., 2001]. The $L s h$ knockout mouse develops fairly normally but dies within a few hours of birth [Geiman et al., 2001]. Additionally, these mice have renal lesions and defects in lymphoid development and proliferation [Geiman and Muegge, 2000; Geiman et al., 2001]. One of the most interesting findings in the study of these mice comes from the discovery that while Lsh -/- mice develop fairly normally until birth, they have global defects in the level of DNA methylation, both in repetitive elements and single copy genes [Dennis et al., 2001]. The substantial loss of methylation in both DDM1 and Lsh mutants indicates that these chromatin remodelers have important roles in the control of genomic DNA methylation levels. Surprisingly, it also appears from these studies that the proper DNA methylation levels controlled by SNF2-like ATPases are not essential until birth. Lsh may be required for accessibility of DNMTs to DNA in chromatin or protection against DNA demethylation. This connection between chromatin remodeling and DNA methylation supports the notion that there are multiple layers of epigenetic modifications to modulate gene expression.

Another member of the SNF2 family of chromatin remodeling proteins is ATRX. This gene is mutated in a human disorder called ATRX (alpha thalassemia and mental retardation, X-linked) syndrome [Gibbons et al., 2000]. The ATRX gene resides on the $\mathrm{X}$ chromosome and when mutated leads to an unusual form of thalassemia, presumably resulting from a $30-$ $60 \%$ decrease in alpha globin gene expression levels [Gibbons et al., 2000]. Although ATRX patients have no alterations in the methylation pattern of the alpha globin gene, there are both hyper and hypomethylation changes in highly repetitive elements such as satellite DNA [Gibbons et al., 2000]. This is in contrast to the defects seen with loss of Lsh in that the total level of 5-methyl cytosine in the genome appears unchanged in ATRX syndrome, while those of $L s h$-/ - mice are dramatically decreased ( $50 \%)$ [Gibbons et al., 2000; Dennis et al., 2001]. Additionally, loss of Lsh leads to hypomethylation only, whereas mutation of $A T R X$ confers both increases and decreases in DNA methylation. A third difference is that only repetitive DNA regions have altered methylation patterns in ATRX, while $L s h-/-$ mice have loss of methylation in single copy genes as well as repeats. These differences could indicate that while both of these chromatin remodeling family members are necessary for proper DNA methylation, the mechanisms may differ, perhaps due to a different complement of interacting proteins.

A recent exciting report demonstrates a link between chromatin remodeling, DNA methylation, and histone H3 lysine 9 methylation [Gendrel et al., 2002]. It had been shown previously that mutation of DDM1 leads to a loss of genomic DNA methylation, particularly in repetitive elements and transposons [Jeddeloh et al., 1999]. In this report, the authors observe that loss of DDM1 function leads to loss of histone $\mathrm{H} 3$ lysine 9 methylation in addition to DNA methylation [Gendrel et al., 2002]. In fact, they observed that heterochromatic histone $\mathrm{H} 3$ methylation patterns change from having predominantly methylated lysine 9 in wild type Arabidopsis strains, to having methylated lysine 4 in mutant DDM1 strains. This data indicates that an SNF2 chromatin remodeling factor such as DDM1 functions prior to, or coincidentally with, histone H3 lysine 
9 methyltransferases, and provides evidence for how the hypomethylated state of genomic DNA in DDM1 mutants is inherited.

\section{MODEL FOR HOW EPIGENETIC FACTORS REGULATE GENE EXPRESSION}

From these observations, we propose a model in which DNA methylation, histone methylation and deacetylation, as well as chromatin remodeling by an SNF2 family protein, cooperate in establishing and maintaining multiple layers of epigenetic gene regulation. In this scheme of how epigenetic mechanisms affect gene expression (Fig. 2), chromatin remodeling and histone modifications act to enable DNMTs to efficiently gain access to the DNA sequence. In our model, histone deacetylation is the first step in the switch from active to repressive chromatin status. As mentioned previously, this step is necessary prior to histone tail methylation at lysine 9, since acetyl groups must be removed from the histone tails. We propose that a chromatin remodeler such as DDM1/Lsh acts next to alter the chromatin structure so that proteins such as HMTase can modify the histone tails, specifically on lysine 9 of histone H3. It has been shown for other chromatin remodeling enzymes that deacetylation of lysines 12 and 16 on histone $\mathrm{H} 4$ greatly facilitates nucleosome mobilization [Clapier et al., 2002] and a similar mechanism may exist for histone H3. The recent report by Gendrel et al. [2002] supports the action of an SNF2 family member preceding the histone methyltransferase step. The DDM1/Lsh chromatin remodeler may function to open the chromatin structure to allow histone and DNA modifying proteins to gain access to the DNA, or may create a DNA/histone configuration that is recognized by DNMTs or their associated proteins. Following this chromatin structure alteration and histone methylation, DNMTs can gain access to the DNA sequence that was previously packaged into an inaccessible confirmation. With the methylation of DNA, other proteins acting alone, or as complexes, can bind the methylated DNA and contribute to a 'tightening' of the chromatin structure thus ensuring long-term and heritable gene silencing. In this scenerio, histone deacetylases may act at several stages of this process-prior to histone H3 lysine 9 methylation, possibly with DNMTs, and finally with the repressive methyl cytosine binding proteins. The requirement of HDAC's at multiple points in the pathway from

\section{Unmethylated DNA}

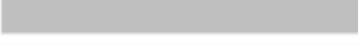
Histone
Histone K9
Methylation
DNA Deacetylation Methylation Methylation
More Chromatin Remodeling ?

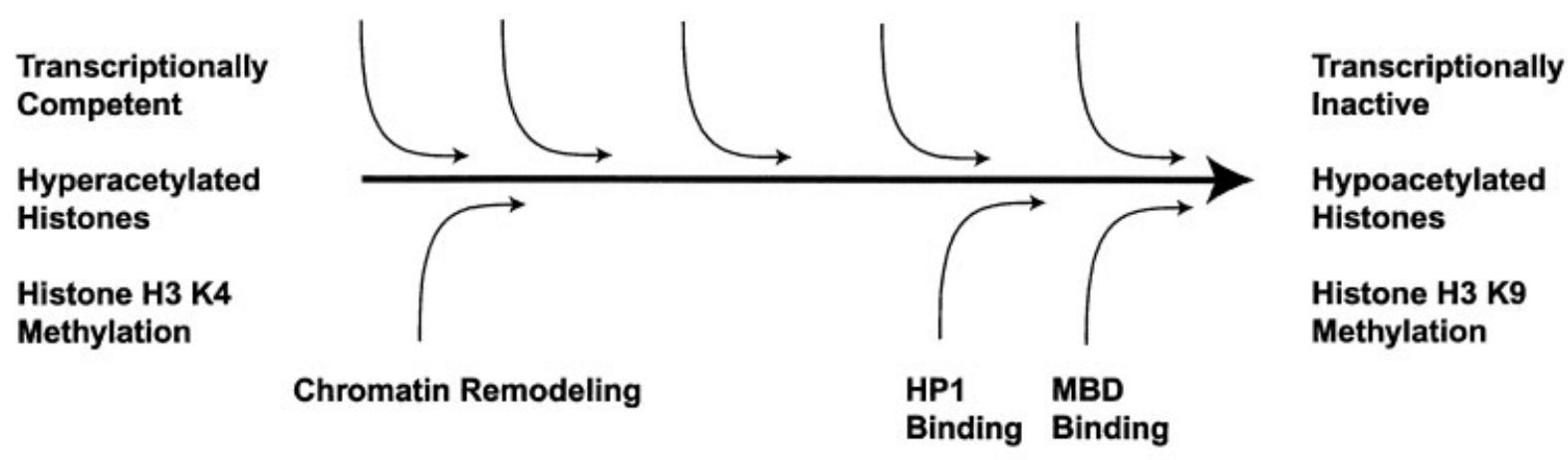

Fig. 2. Model for how multiple epigenetic modifications can convert unmethylated, "open" chromatin into methylated, "closed" chromatin. Arrows indicate the possible sites of each epigenetic action. HP1, heterochromatin protein 1; MBD, methyl-CpG-binding domain protein. 
transcriptional competence to silence is further emphasized by the findings that HDAC's interact with all major components of the pathway, HMTase (SUV39H1), DNMT, and chromatin remodeling enzyme [Vignali et al., 2000; Czermin et al., 2001; Nakayama et al., 2001; Clapier et al., 2002; Robertson, 2002] (Tables I and II). Additionally, one or more SNF2 family members may act to allow proteins such as histone deacetylases, histone methylases, and DNMTs to gain access to the chromatin, as well as function in the establishment of a compact, repressive chromatin state. As a final step in maintaining a repressive chromatin state such as that seen in heterochromatin, HP1, and perhaps methyl-CpG-binding proteins such as MeCP2 or the NuRD complex, may bind the altered chromatin structure and reinforce transcriptional silencing.

It is clear that these exciting new observations point to the importance of multiple layers of epigenetic modifications in the control of chromatin structure and gene expression. Future studies will be needed to elucidate the complex interplay between these various epigenetic mechanisms. Specifically, a better understanding of how these modifications are targeted to specific regions of the genome, how they are altered during the cell cycle and upon differentiation, and how these signals can be manipulated pharmacologically will be essential. Additionally, investigation into the proteins that interact with DNMTs, HMTase, and chromatin remodelers will aid in better understanding the regulation of these enzymes (Table II). Finally, it will be important to determine whether these epigenetic modulators directly interact with one another or whether their connection is indirect. The area of epigenetics has surely become much more complex in recent years, but investigation of this complexity has the potential to shed new light on the fundamentals of gene regulation and chromatin structure, and its deregulation as it pertains to inherited human disorders and cancer.

\section{ACKNOWLEDGMENTS}

K.D. Robertson is a Cancer Scholar supported by the National Cancer Institute (NIH grant CA84535-01). T.M. Geiman is a Pharmacology Research Associate Training (PRAT) Fellow supported by the National Institute of General Medical Sciences, NIH. We apologize to those whose work we were not able to cite directly due to limited space.

\section{REFERENCES}

Aoki A, Suetake I, Miyagawa J, Fujio T, Chijiwa T, Sasaki H, Tajima S. 2001. Enzymatic properties of de novo-type mouse DNA (cytosine-5) methyltransferases. Nucleic Acids Res 29:3506-3512.

Bachman KE, Rountree MR, Baylin SB. 2001. Dnmt3a and Dnmt3b are transcriptional repressors that exhibit unique localization properties to heterochromatin. J Biol Chem 276:32282-32287.

Bannister AJ, Zegerman P, Partridge JF, Miska EA, Thomas JO, Allshire RC, Kouzarides T. 2001. Selective recognition of methylated lysine 9 on histone $\mathrm{H} 3$ by the HP1 chromo domain. Nature 410:120-124.

Baylin S, Jones PA. 2002. The fundamental role of epigenetic events in cancer. Nat Rev Genet 3:415-428.

Bestor TH. 2000. The DNA methyltransferases of mammals. Hum Mol Genet 9:2395-2402.

Clapier CR, Nightingale KP, Becker PB. 2002. A critical epitope for substrate recognition by the nucleosomal remodeling ATPase ISWI. Nucleic Acids Res 30:649655.

Czermin B, Schotta G, Hulsmann BB, Brehm A, Becker PB, Reuter G, Imhof A. 2001. Physical and functional association of SU(VAR)3-9 and HDAC1 in Drosophila. EMBO Rep 2:915-919.

Dennis K, Fan T, Geiman T, Yan Q, Muegge K. 2001. Lsh, a member of the SNF2 family, is required for genome-wide methylation. Genes Dev 15:2940-2944.

Ehrlich M, Buchanan KL, Tsien F, Jiang G, Sun JB, Uicker W, Weemaes CMR, Smeets D, Sperling K, Belohradsky BH, Tommerup N, Misek DE, Rouillard J-M, Kuick R, Hanash SM. 2001. DNA methyltransferase 3B mutations linked to the ICF syndrome cause dysregulation of lymphogenesis genes. Hum Mol Genet 10:2917-2931.

Geiman TM, Muegge K. 2000. Lsh, an SNF2/helicase family member, is required for proliferation of mature $\mathrm{T}$ lymphocytes. Proc Natl Acad Sci USA 97:4772-4777.

Geiman TM, Tessarollo L, Anver MR, Kopp JB, Ward JM, Muegge K. 2001. Lsh, a SNF2 family member, is required for normal murine development. Biochim Biophys Acta 1526:211-220.

Gendrel A-V, Lippman Z, Yordan C, Colot V, Martienssen R. 2002. Dependence of heterochromatic histone H3 methylation patterns on the Arabidopsis gene DDM1. Science 20 June, online 10.1126 .

Gibbons RJ, McDowell TL, Raman S, O'Rourke DM, Garrick D, Ayyub H, Higgs DR. 2000. Mutations in ATRX, encoding a SWI/SNF-like protein, causes diverse changes in the pattern of DNA methylation. Nat Genet 24:368-371.

Hansen RS, Wijmenga C, Luo P, Stanek AM, Canfield TK, Weemaes CMR, Gartler SM. 1999. The DNMT3B DNA methyltransferase gene is mutated in the ICF immunodeficiency syndrome. Proc Natl Acad Sci USA 96:1441214417.

Hata K, Okano M, Lei H, Li E. 2002. Dnmt3L cooperates with the Dnmt3 family of de novo DNA methyltransferases to establish maternal imprints in mice. Development 129:1983-1993. 
Havas K, Whitehouse I, Owen-Hughes T. 2001. ATPdependent chromatin remodeling activities. Cell Mol Life Sci 58:673-682.

Hsieh C-L. 1999. In vivo activity of murine de novo methyltransferases, Dnmt3a and Dnmt3b. Mol Cell Biol 19:8211-8218.

Jackson JP, Lindroth AM, Cao X, Jacobsen SE. 2002. Control of $\mathrm{CpNpG}$ methylation by the KRYPTONITE histone H3 methyltransferase. Nature 416:556-560.

Jarvis CD, Geiman T, Vila-Storm MP, Osipovich O, Akella U, Candeias S, Nathan I, Durum SK, Muegge K. 1996. A novel putative helicase produced in early murine lymphocytes. Gene 169:203-207.

Jeddeloh JA, Stokes TL, Richards EJ. 1999. Maintenance of genomic methylation requires a SWI2/SNF2-like protein. Nat Genet 22:94-97.

Lachner M, Jenuwein T. 2002. The many faces of histone lysine methylation. Curr Opin Cell Biol 14:286-298.

Lachner M, O'Carroll D, Rea S, Mechtler K, Jenuwein T. 2001. Methylation of histone H3 lysine 9 creates a binding site for HP1 proteins. Nature 410:116-120.

Lei H, Oh SP, Okano M, Juttermann R, Goss KA, Jaenisch R, Li E. 1996. De novo DNA cytosine methyltransferase activities in mouse. Development 122:3195-3205.

Leonhardt H, Page AW, Weier H, Bestor TH. 1992. A targeting sequence directs DNA methyltransferase to sites of DNA replication in mammalian nuclei. Cell 71: 865-873.

Li E, Bestor TH, Jaenisch R. 1992. Targeted mutation of the DNA methyltransferase gene results in embryonic lethality. Cell 69:915-926.

Miura A, Yonebayashi S, Watanabe K, Toyama T, Shimada H, Kakutani T. 2001. Mobilization of transposons by a mutation abolishing full DNA methylation in Arabidopsis. Nature 411:212-214.

Nakayama J, Rice JC, Strahl BD, Allis CD, Grewal SIS. 2001. Role of histone H3 lysine 9 methylation in epi genetic control of heterochromatin assembly. Science 292:110-113.

Okano M, Xie S, Li E. 1998. Cloning and characterization of a family of novel mammalian DNA (cytosine-5) methyltransferases. Nat Genet 19:219-220.

Okano M, Bell DW, Haber DA, Li E. 1999. DNA methyltransferases Dnmt3a and Dnmt3b are essential for de novo methylation and mammalian development. Cell 99:247-257.

Raabe EH, Abdurrahman L, Behbehani G, Arceci RJ. 2001. An SNF2 factor involved in mammalian development and cellular proliferation. Dev Dyn 221:92105.

Rea S, Eisenhaber F, O'Carroll D, Strahl BD, Sun Z-W, Schmid M, Opravil S, Mechtler K, Ponting CP, Allis CD, Jenuwein T. 2000. Regulation of chromatin structure by site-specific histone H3 methyltransferases. Nature 406:593-599.

Reik W, Dean W, Walter J. 2001. Epigenetic reprogramming in mammalian development. Science 293:10891093.

Robertson KD. 2002. DNA methylation and chromatinunraveling the tangled web. Oncogene (in press).

Tamaru H, Selker EU. 2001. A histone H3 methyltransferase controls DNA methylation in Neurospora crassa. Nature 414:277-283.

Vignali M, Hassan AH, Neely KE, Workman JL. 2000. ATP-dependent chromatin-remodeling complexes. Mol Cell Biol 20:1899-1910.

Wang H-B, Cao R, Xia L, Erdjument-Bromage H, Borchers C, Tempst P, Zhang Y. 2001. Purification and functional characterization of a histone H3-lysine 4-specific methyltransferase. Mol Cell 8:1207-1217.

Yokochi T, Robertson KD. 2002. Preferential methylation of unmethylated DNA by mammalian de novo DNA methyltransferase Dnmt3a. J Biol Chem 277:1173511745. 